\title{
Rice Grain Physical and Chemical analysis from Paddy Drying by Using a Solar Tunnel Dryer at Prathum Tani, Thailand
}

\author{
Krissadang Sookramoon ${ }^{1}$ and Mananya Khamwachirapithak ${ }^{2}$ \\ ${ }^{1}$ Faculty of Industrial Technology Vallaya Alongkorn Rajabhat University 1 Moo 20 Tambol \\ Klongnueng Amphoe Klongluang PathumThani, Thailand \\ ${ }^{2}$ Faculty of Science and Technology Vallaya Alongkorn Rajabhat University 1 Moo 20 Tambol \\ Klongnueng Amphoe Klongluang PathumThani, Thailand
}

\begin{abstract}
This paper presents the RD31 rice grain physical and chemical analysis from paddy drying by using a solar tunnel dryer. RD31 paddy was dried in a compact solar tunnel dryer, with a drying chamber volume in the size of $0.113 \mathrm{~m}^{3}$ and $2.112 \mathrm{~m}^{2}$ flat plate collectors. The experiment was performed on May 15, 2014 at Faculty of Industrial Technology Vallaya Alongkorn Rajabhat University Pathum Tani Thailand. The samples for testing were three varieties of Thai rice namely RD31 (from the farm), RD31 (dried in a solar tunnel dryer), and Prathum Tani 1 (from the market). The test consists of RD 31 dried by a solar tunnel dryer; the moisture content was reduced to about 14 wt. \%. Then 3 varieties of rice were milled and sent to the laboratory for analyzing physical and chemical properties, repeated 3 times. The results showed that the average drying temperature was $67.75{ }^{\circ} \mathrm{C}$. The paddy moisture reduction content was estimated from 49.96 to $14.64 \mathrm{MC}$ (\% w.b.) within 6 hours. All dimensions of RD31, dried by using solar tunnel dryer milled rice were closely related to those of the Prathum Tani 1, purchased from the market. The ratio of length to width of 3 samples: RD31 (raw rice), RD31 (dried in solar tunnel dryer), and Prathum Tani 1 (from the market) were $3.54,3.47$, and 3.44 , respectively. 3 rice grain varieties were slender. The yield percentage was $60 \%, 75 \%$, and $70 \%$, respectively. Results from chemical test showed that the alkaline spreading value were $4-5$, 3 , and 2, respectively. The quality of the RD31 rice grain dried in a solar tunnel dryer passed the Thailand Ministry of Agriculture rice quality standard
\end{abstract}

\section{Introduction}

Thailand, one of the rice exporter countries in the world produces high quality rice such as RD31 Thai rice. Rice can be used for producing many products such as rice flour, rice oil, and many food applications. The quality of drying paddy is important for producing high quality rice. Rice quality is a combination of physical and chemical characteristics. Some studies of solar dryer for paddy drying have been reported. Excell and Kornsakoo [1] designed a cheap efficiently solar dryer for paddy drying namely AIT (Asian Institute of Technology) dryer in Thailand. It consists of the solar collector, paddy drying chamber, and the exhaust air chimney. Suharwadji and Takiyah [2] evaluated AIT dryer in Indonesia. 100 kilograms of paddy were dried in $76 \mathrm{~h}$ with a range of the moisture content of $22-10 \%$ and concluded that there was no difference between this method and traditional sun drying method. On and Than [3] developed a paddy dryer with an intermittent batch in Malaysia. Centrifugal blower was used to move hot air from the solar collector to dry the products. Harahap and Mustadjab [4] tested a cabinet type solar dryer for paddy drying in Indonesia. $200 \mathrm{~kg}$ Paddy was dried from moisture content of $2224 \%$ to 14 -
$15 \%$ within 3 days. Tongprasert et al. [5] reported the experiment of a forced convection paddy solar dryer. The dryer with a $3.74 \times 4.48 \mathrm{~m}^{2}$ flat plate solar collector and a drying bin showed that $1,000 \mathrm{~kg}$ of paddy could be dried in 1-4 days from a moisture content of $17.21-14 \%$. The objective of this work is to investigate the RD31 rice grain physicochemical properties from RD31paddy drying by using a solar tunnel dryer and compare the product quality with $\mathrm{RD} 31$, a product of the tradition sun drying method (14 wt.\% moisture content).

\section{Materials and methods}

\subsection{The Solar Tunnel Dryer for paddy drying}

This work focuses on rice grain physical and chemical analysis from paddy drying at Prathum Tani, Thailand. The paddy is sent to the solar tunnel dryer for drying. The paddy is loaded into the drying chamber of the solar tunnel dryer [6]. Hot air, which was obtained from $14 \mathrm{~W}$, $1000 \mathrm{ft}^{3} / \mathrm{min}$ DC electric fan, is circulated and passed through the RD31 paddy in drying chamber and the paddy dry. The moist air is passed out through the exhaust after drying. The door is opened and RD31 
paddies are taken out of the tunnel and new batches of wet paddies are introduced into drying chamber for repetition. The solar tunnel dryer, is 2.99 meters long and 1.77 meters wide, with a flat plate solar collector area of $2.112 \mathrm{~m}^{2}$ and a drying chamber volume of $0.113 \mathrm{~m}^{3}$. The paddy drying test was started from 9:00 A.M. to 3.00 P.M. on May 15, 2014. The paddy samples were polished and sent to analyze the physical and chemical properties in a laboratory. The dried paddy was weighed for moisture content collection. The initial and final moisture contents of the product before and after each day of drying were determined. The solar radiation data and other meteorological data were obtained from the Mechanical Technology Workshop Station at Vallaya Alongkorn Rajabhat University Pathum Tani. Fig. 1 shows Solar Tunnel Dryer.

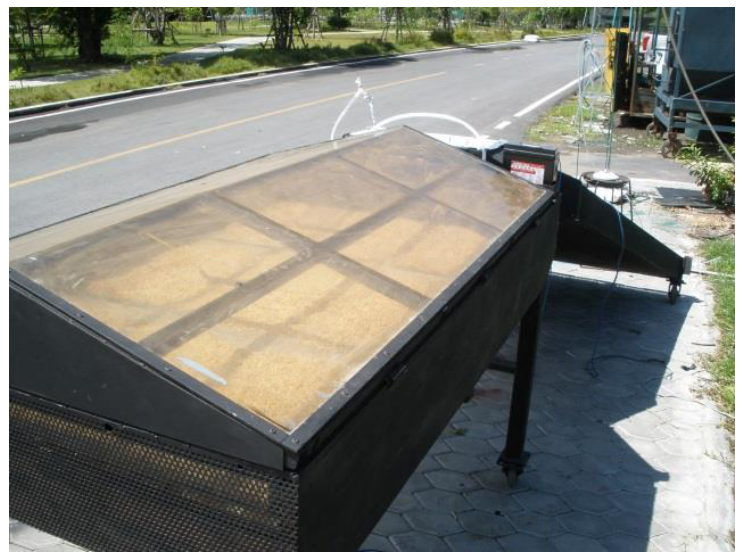

Figure 1. The solar tunnel dryer with paddy drying.

\subsection{Samples preparation}

3 milled rice grain samples: RD31 (raw rice from farm in Prathum Tani Province), RD31 (dried in a solar tunnel dryer), and Prathum Tani 1 (purchased from the market) with approximately $14 \%$ moisture content were collected and prepared. This work was carried out in the Laboratory Department of Food Science and Technology in Vallaya Alongkorn Rajabhat University, Thailand. The grains were cleaned to remove all substances. The length, width and slenderness were measured using a digital vernier caliper ( $0.001 \mathrm{~mm}$ accuracy). Grain mass was measured with a sensitive electronic balance $(0.001 \mathrm{gm}$ sensitivity). After the determination of the dimensions, the grain density was determined using a beaker and electronic balance. The bulk density was determined with a weight (gram) per milliliter. Then the rice grain color measurement and chemical properties were measured.

\subsection{Physical characteristics of rice grain}

Grain quality physical examination generally includes dimensions, shape, color, percentage of the rice mixture, and density. Grain Dimensions were measured with a digital vernier caliper. Measurements are in millimeters. Grain dimensions are used to separate the rice into three categories; short, medium, or long grains.
1. Rice grain dimensions measurements

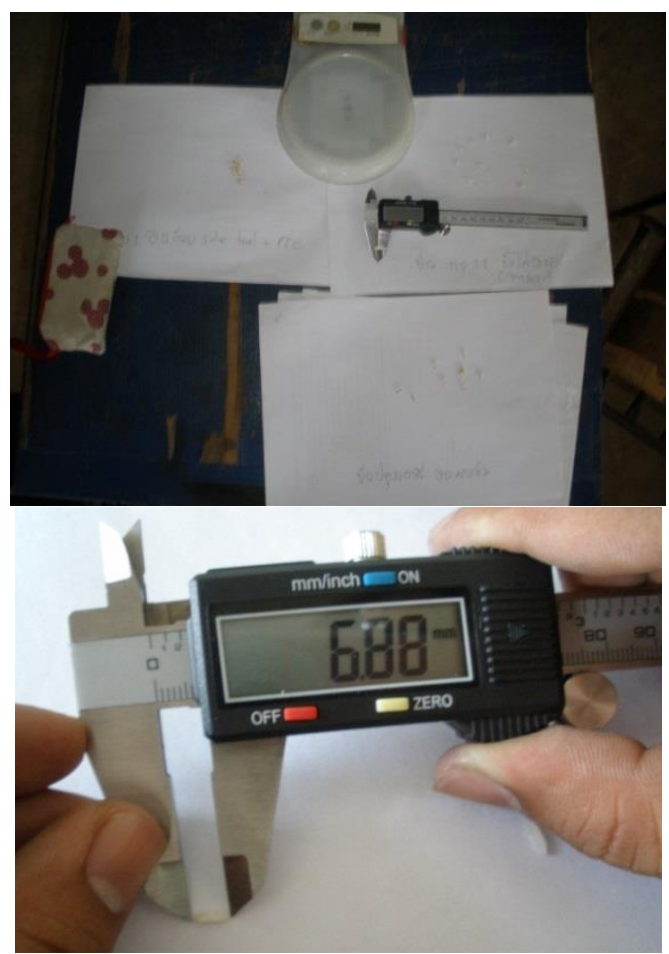

Figure 2. Equipment for measuring the size of a rice grain, including an electronic balance, a digital vernier caliper, and a digital camera.

\section{Rice grain color measurement}

The rice grain color meter was used to determine rice grain color. The obtained value was evaluated by using a Minolta chart as shown in Fig. 3 below.

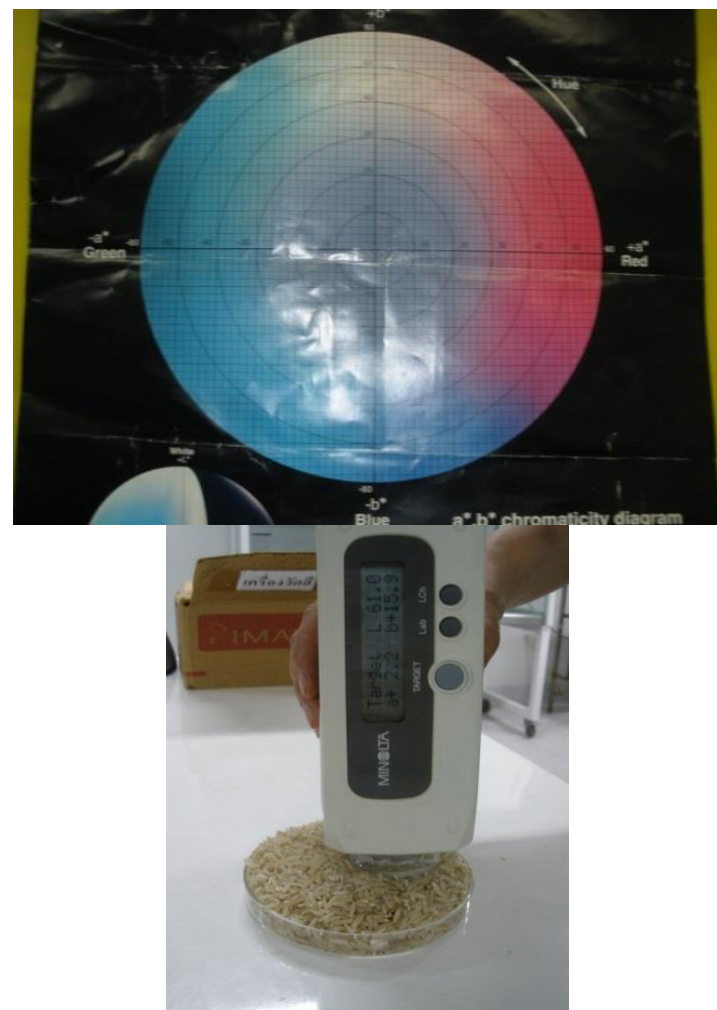

Figure 3. Minolta chart for rice grain color reading and a color meter for measuring the rice grain color. 


\subsection{Chemical characteristics of rice grain (Alkali Spreading Value)}

The purpose of this technique is to asset the gel type of the rice, which indicates the gelatinization temperature $\left(60-70^{\circ} \mathrm{C}\right)$. The first step was preparing a solution of potassium hydroxide in a concentration of $1.7 \mathrm{wt}$. \% $(0.05 \%)$. Consider the dissolution of the grain in each seed spotted by a breakdown in Table 4 .

\section{Results and discussion}

Table 1. Results of rice color evaluation

\begin{tabular}{|l|l|l|}
\hline Order & Type of rice grain & Color Evaluation \\
\hline 1 & RD31 (raw rice) & No.1 L70.0 a $+1.5 \mathrm{~b}+13.4$ \\
& & No.2 L65.8 a+0.9 b+12.4 \\
& & No.3 L68.9 a+1.1 b+13.8 \\
& & Ave. L68.23 a+1.16 b+13.2 \\
\hline 2 & RD31 (dried in solar & No.1 L61.4 a $+2.5 \mathrm{~b}+15.4$ \\
& No.2 L61 a $+2.2 \mathrm{~b}+15.9$ \\
& & No.3 L62.4 a+2.0 b+14.7 \\
& & Ave. L61.6 a+2.23 b+15.33 \\
\hline 3 & Pratum Tani 1 (dried and & No.1 L68.3 a $+4.1 \mathrm{~b}+18.7$ \\
& purchased from the & No.2 L64.9 a+0.6 b+19.3 \\
& market) & No.3 L64.2 a $+3.5 \mathrm{~b}+20$ \\
& & Ave. L65.8 a+2.73 b+19.33 \\
\hline
\end{tabular}

$\mathrm{L}=$ brightness $\mathrm{a}=$ red color $\mathrm{b}=$ yellow

Table 2. The average length and width of a grain of rice

\begin{tabular}{|c|c|c|c|c|c|c|c|c|}
\hline \multirow{2}{*}{ No. } & \multicolumn{5}{|c|}{ Length (mm) } & \multicolumn{3}{c|}{ Width (mm) } \\
\cline { 2 - 9 } & 1 & 2 & 3 & Ave. & 1 & 2 & 3 & Ave. \\
\hline RD31 (raw rice) & 6.88 & 7.15 & 6.81 & 6.946 & 1.94 & 2.02 & 1.93 & 1.963 \\
\hline $\begin{array}{c}\text { RD31 (dried in } \\
\text { solar tunnel dryer) }\end{array}$ & 7.05 & 6.76 & 6.8 & 6.87 & 2.07 & 1.87 & 2.01 & 1.983 \\
\hline $\begin{array}{c}\text { Pratum Tani 1 } \\
\text { from market) }\end{array}$ & 7.5 & 7.32 & 7.51 & 7.443 & 2.14 & 2.61 & 1.87 & 2.206 \\
\hline
\end{tabular}

Table 3. Grain shape, length / width of a grain

\begin{tabular}{|c|c|c|c|c|}
\hline Type of rice grain & \multicolumn{4}{|c|}{ Grain shape, length/width of a grain } \\
\cline { 2 - 5 } & 1 & 2 & 3 & Ave. \\
\hline RD31 (raw rice) & 3.55 & 3.54 & 3.53 & 3.54 \\
\hline $\begin{array}{c}\text { RD31 (dried in solar } \\
\text { tunnel dryer) }\end{array}$ & 3.4 & 3.61 & 3.38 & 3.47 \\
\hline $\begin{array}{c}\text { Pratum Tani 1 (from } \\
\text { market) }\end{array}$ & 3.5 & 2.8 & 4.02 & 3.44 \\
\hline
\end{tabular}

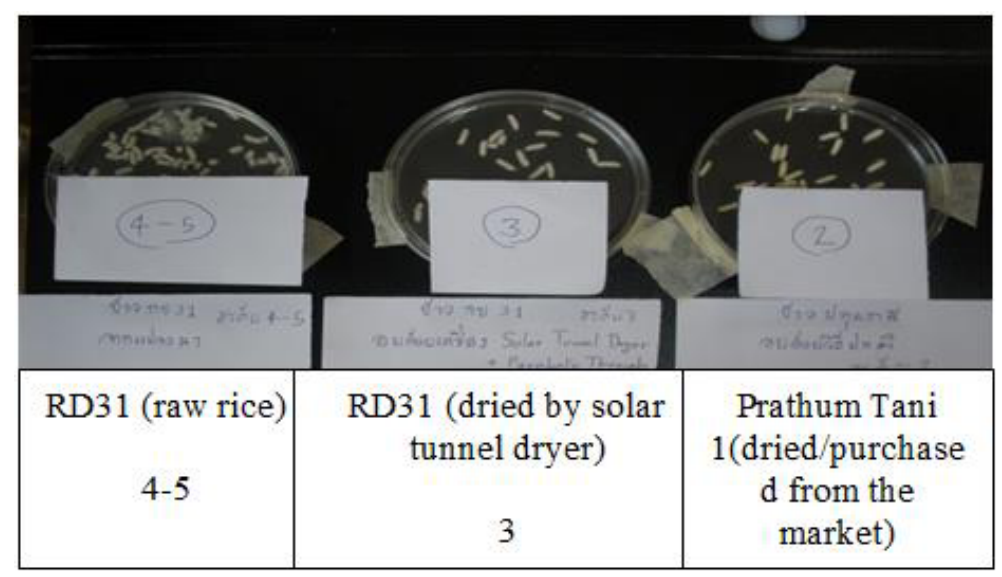

Figure 4. The alkali spreading values 
Table 4. The alkali spreading value

\begin{tabular}{|l|c|l|}
\hline Type of rice grain & Alkali Spreading Value & \multicolumn{1}{c|}{ Description } \\
\hline RD31 (raw rice) & $4-5$ & $\begin{array}{l}\text { The surface of the transverse or } \\
\text { longitudinal seams grain and flour spread } \\
\text { out of the seeds over a wide area. }\end{array}$ \\
\hline $\begin{array}{l}\text { RD31 (dried in a solar tunnel } \\
\text { dryer) }\end{array}$ & 3 & $\begin{array}{l}\text { Seeds, puffed rice and flour spread from } \\
\text { some parts of the grain. }\end{array}$ \\
\hline $\begin{array}{l}\text { Prathum Tani 1 (purchased } \\
\text { from the market) }\end{array}$ & 2 & Seeded rice crispiest \\
\hline
\end{tabular}

\section{Summary}

The physical and chemical analysis from paddy drying at Prathum Tani, using solar tunnel dryer was experimental study. The results clearly indicate that the average drying temperature was $67.75{ }^{\circ} \mathrm{C}$. The RD31 paddy moisture content was reduced from $49.96-14.64 \mathrm{MC}$ (\% w.b.) in 6 hours. All dimensions of RD31, dried by using solar tunnel dryer milled rice were closely related to those of the Prathum Tani 1, which was purchased from the market. The ratio of length to width of 3 samples: RD31 (raw rice), RD31 (dried in solar tunnel dryer), and Prathum Tani 1 (from the market) were 3.54, 3.47, and 3.44 , respectively. Three rice grain varieties were slender. The yield percentage was $60 \%, 75 \%$, and $70 \%$, respectively. Results from chemical test showed that the alkaline spreading value were $4-5,3$, and 2, respectively. The quality of paddy dried in this solar tunnel dryer has the same quality as a conventional drying technique, but could reduce the time of drying in one third.

\section{Acknowledgement}

The authors would like to acknowledge the Research and Development Institute of Rajabhat Vallaya Alongkorn University for financial support for this research.

\section{References}

1. Excell RHB, Kornsakoo S, A low cost solar rice dryer, Journal of Approp Technol, 5(1) 23-24, (1987)

2. Suharwadji, Takiyah, Kamar Pengering Model EXELL, Technical Report, Lembaga Fisika Nasional, Bandung, Indonesia, (1983)

3. Ong KS, Than CF, A forced convection solar paddy dryer, Bulletin, July, Institution of Engineers Malaysia, (1982)

4. Harahap F, Suwono A, Abdurrachim Sapardi J, Prayudi, A Description and preliminary test results of a two ton solar paddy dryer. In Proceedings of regional Asia and Pacific workshop on the applications of solar energy in agricultural and postharvest activities, Bandung, Indonesia, (1981)

5. Thanvi KP, Pandey PC Development of low cost solar Agricultural dryer for regions of India energy in agricultural dryer for arid regions of India, Journal of Energy Agri. 6, 35-40, (1987)

6. Krissadang Sookramoon, Performance Evaluation of a Solar Tunnel Dryer for Paddy Drying at Prathum Tani, Thailand, Journal of App Mec and Mats, 799800, 1455-1458, (2015) 